\title{
Recent advances in the theory of disordered systems ${ }^{\star}$
}

\author{
Ferenc Iglói ${ }^{1,2, a}$ and Heiko Rieger ${ }^{3}$ \\ ${ }^{1}$ Wigner Research Centre for Physics, Institute for Solid State Physics and Optics, 1525 Budapest, P.O. Box 49, \\ Hungary \\ ${ }^{2}$ Institute of Theoretical Physics, Szeged University, 6720 Szeged, Hungary \\ 3 Theoretical Physics, Saarland University, 66123 Saarbrücken, Germany
}

Received 19 March 2020

Published online 14 May 2020

(C) EDP Sciences / Società Italiana di Fisica / Springer-Verlag GmbH Germany, part of Springer Nature, 2020

Real materials always contain some kind of quenched disorder: in regular lattices these are point defects, substitutional atoms or dislocations; structural disorder is present in glasses, liquid mixtures and macromolecules; magnetic materials with random lattices and/or random, position dependent interactions establish the classes of random magnets and spin glasses; and experiments with ultracold atoms can be designed to include disorder in a controllable way.

The theory of disordered systems is multifaceted and comprises various topics of fundamental importance: the localization of electronic states in non-interacting systems and many body localization in the presence of interactions, quantum phase transitions in disordered quantum many body systems, dynamical heterogeneities in spin glasses and glassy materials, phase transitions in random ferromagnets and random field systems, to name but a few. Due to the broad range of problems monographs presenting a comprehensive picture of all facets of disordered systems are still lacking. In the second part of the last century J.M. Ziman made an effort to lay down the basic notations about the theory of disordered systems in his book The models of disorder [1]. However several modern developments were missing in this treatment, as for instance Parisi's solution of the Sherrington-Kirkpatrick spin-glass model [2-4], the scaling theory of Anderson localisation [5], the field theory of phase transitions in random systems [6], the infinite disorder scaling $[7,8]$ and many more. The theory of disordered systems is still a notoriously difficult discipline mainly due to the fact that exactly solved non-trivial problems, which could serve as guidance to validate approximate and numerical methods, are scarce.

In this special issue: Recent Advances in the Theory of Disordered Systems original research articles, mini-reviews and pedagogical introductions are collected

\footnotetext{
* Contribution to the Topical Issue "Recent Advances in the Theory of Disordered Systems", edited by Ferenc Iglói and Heiko Rieger.

a e-mail: igloi.ferenc@wigner.hu
}

from different areas of research. A broad range of topics is covered in this issue including classical and quantum systems, methodological papers, numerical and analytical computations. In the following we shortly summarize the subject of the different contributions.

\section{Classical disordered systems}

This section starts with a review by Tarjus and Tissier about the critical behaviour of random field Ising and $O(N)$ models recent results obtained from the functional renormalization group method. This approach solved questions that were pending for many years, such as the mechanism for the breakdown of dimensional reduction and the breaking of the underlying supersymmetry below $d=6[9]$.

Next Kumar, Corberi, Lipiello and Puri numerically studied the ordering kinetics in a two-dimensional Ising model in which the fraction of antiferromagnetic links $a$ is tuned gradually. They found that, upon increasing $a$, the behavior changes in a radical way. Small $a$ does not prevent the system from a complete ordering, but this occurs in an extremely (logarithmically) slow manner. However, larger values of this parameter destroy complete ordering, due to frustration, and the evolution is comparatively faster (algebraic) [10].

In another paper Delfino and Lamsen presented a field theoretical study of the disorder rounded first-order transition in the $Q>4$ state random bond Potts model. Within the recently introduced exact framework of scale invariant scattering they have found the line of stable fixed points induced by disorder for arbitrarily large values of $Q[11]$.

Finally, Cocco, Croce and Zamponi used the Adaptive Cluster Expansion method to study numerically the direct Ising problem, that is, to compute the free energy and the equilibrium observables of spin systems with arbitrary two-spin interactions. The properties of the cluster expansion and its performance are studied in detail for one dimensional, two dimensional, random and fully connected graphs with homogeneous or heterogeneous fields and couplings [12]. 


\section{Computer simulations}

Computer simulations of systems with quenched disorder are extremely demanding, suffering from the combined effect of slow relaxation and the need of performing the disorder average. As a consequence, new algorithms, improved implementations, and alternative and even purpose-built hardware are often instrumental for conducting meaningful studies of such systems. The ensuing demands regarding hardware availability and code complexity are substantial and sometimes prohibitive. Kumar, Gross, Janke and Weigel demonstrated how with a moderate coding effort leaving the overall structure of the simulation code unaltered as compared to a CPU implementation, very significant speed-ups can be achieved from a parallel code on GPU by mainly exploiting the trivial parallelism of the disorder samples and the neartrivial parallelism of the parallel tempering replicas [13]. Schnabel and Janke have described how metastable states of Ising spin glasses can be counted by means of Monte Carlo computer simulations. The method is applied to systems defined on hypercubic lattices in one to six dimensions with up to about $10^{3}$ spins. It is shown that the number of metastable states obtained for different disorder realizations satisfies a log-normal distribution. The distribution of energies of metastable states by means of moments and cumulants are also investigated [14].

\section{Quantum phase transitions and critical systems}

A relatively large portion of this topical issue deals with quantum disordered systems. Many contributions in this section focus on a class of random quantum systems the quantum critical properties are controlled by a so called infinite disorder fixed point, at which disorder fluctuations are dominant over quantum fluctuations. Such systems are conveniently studied by the strong disorder renormalization group (SDRG) method, which is expected to provide asymptotically exact results for large scales.

The SDRG method formulated in terms of a tree tensor network is used by Tsai, Chen and Lin to study spin-1 random Heisenberg antiferromagnetic chains. As the randomness strength is increased a phase transition is identified between a gapless Haldane phase and a critical random-singlet phase. They have determined the critical exponents related to the average string order parameter, the average end-to-end correlation function and the average bulk spin-spin correlation function, both at the critical point and in the random-singlet phase [15].

Using the SDRG method Quito, Lopes, Hoyos and Miranda have shown that strongly disordered spin chains invariant under the $S O(N)$ group display random-singlet phases with emergent $S U(N)$ symmetry without fine tuning. The phases with emergent $S U(N)$ symmetry are of two kinds: one has a ground state formed of randomly distributed singlets of strongly bound pairs of $S O(N)$ spins (a 'mesonic' phase), while the other has a ground state composed of singlets made out of strongly bound integer multiples of $N, S O(N)$ spins (a 'baryonic' phase) [16].

Getelina and Hoyos studied the spin-spin correlations in two distinct random critical XX spin- $1 / 2$ chain models via exact diagonalization. While the exponents of the transverse typical and mean correlations are found nearly equal, implying a narrow distribution of transverse correlations, the longitudinal typical and mean correlations critical exponents are quite distinct implying much broader distributions [17].

Zhang and Rieger studied the phase diagram of the twodimensional Bose-Hubbard model with cavity-mediated long-range interactions and uncorrelated diagonal disorder and compared to that of the extended Bose-Hubbard model with nearest-neighbor interactions in the presence of uncorrelated diagonal disorder. They show that two kinds of Bose glass phases exist: one with and one without density wave order and also find that weak disorder enhances the supersolid phase [18].

Roósz, Kovács and Iglói studied the entanglement entropy of such subsystems, which has a linear extent $L$ and contains a fraction of randomly selected points $p$, in one- and two-dimensional critical fermionic systems. The leading contribution to the average entanglement entropy is found to scale with the volume, to which there is a logarithmic correction term, $b(p) L^{D-1} \ln L$. In $2 D$ the prefactor has a different functional form of $p$ below and above the percolation threshold [19].

\section{Localization/Anderson transition and energy spectra}

Contributions in this section focus the localization and the Anderson transition disordered quantum systems by analyzing the eigenstates of the Hamiltonian defining the system.

Using numerical simulations Slevin, Kettemann and Ohtsuki investigated the distribution of Kondo temperatures at the Anderson transition. In agreement with previous work, they find that the distribution has a long tail at small Kondo temperatures and checked the validity of a recent theoretical prediction in three dimensions [20].

Eleuch and Hilke studied one-dimensional localisation in the presence of a random potential characterized by an arbitrary autocorrelation function. They calculated the localization length of wave solutions beyond the Born approximation and compared it with numerical results [21].

Kim and Han used the framework of Boltzmann transport theory to investigate metal-insulator transitions at finite temperatures. They focus on two dimensional metalinsulator transitions and compare them with those in three dimensions, considering the diffusion constant as a function of both disorder strength and bath temperature. Their result implies that the role of dephasing in renormalization of the diffusion constant is more complex in two dimensions than that in three dimensions [22].

Thomson and Schiró used flow equation methods to study localisation in disordered quantum systems, and particularly to obtain the non-equilibrium dynamical evolution of observables. They show how this method can be used to compute quench dynamics of simple observables, demonstrate how this formalism provides a natural framework to understand operator spreading and show how to construct complex objects such as correlation functions [23]. 
Chávez, Mattiotti, Méndez-Bermúdez, Borgonovi and Celardo presented a comparison between the single particle spectrum of the discrete Bardeen-Cooper-Schrieffer (BCS) model, used for small superconducting grains, and the spectrum of a paradigmatic model of Single Excitation Superradiance (SES). They are both characterized by an equally spaced energy spectrum (Picket Fence) where all the levels are coupled between each other by a constant coupling which is real for the BCS model and purely imaginary for the SES model. They show that the transition to a Superradiant regime can be connected to the emergence of an imaginary energy gap, similarly to the transition to a Superconductive regime where a real energy gap emerges [24].

In a related context Skipetrov studied the density of states (DOS) in band gaps of ideal and disordered threedimensional photonic crystals of finite size. The DOS inside a band gap of the ideal crystal is found to decrease as the inverse of the crystal size and disorder narrows the band gap and DOS exhibits enhanced fluctuations near the new band edges. However, the average DOS still exhibits the same scaling with the crystal size within the remaining band gap. A phenomenological explanation of this scaling suggests that it should hold for one- and two-dimensional photonic crystals as well [25].

\section{Other problems}

Luck has studied stochastic games exhibiting Parrando's paradox, in which two rules, $\mathrm{A}$ and $\mathrm{B}$, are played at discrete time steps, following either a periodic pattern or an aperiodic one, be it deterministic or random. These games are mapped onto 1D random walks and calculating the gain involves products of non-commuting Markov matrices, which are somehow analogous to the transfer matrices used in the physics of 1D disordered systems. One of the most original sides of this work is the identification of weak-contrast regimes for capital-dependent and history-dependent Parrondo games, and a detailed quantitative investigation of the gain in the latter scaling regimes [26].

Finally Monthus presents a pedagogical introduction to the theory of large deviations in the field of disordered systems. Various emblematic classical and quantum disordered systems are considered in order to highlight the unified perspective the typical events and the rare events that occur on various scales [27].

\section{References}

1. J.M. Ziman, The models of disorder (Cambridge University Press, Cambridge, 1979)

2. G. Parisi, Phys. Lett. A 73, 203 (1979)

3. G. Parisi, J. Phys. A 13, L115 (1980)

4. G. Parisi, J. Phys. A 13, 1101 (1980)

5. E. Abrahams, P.W. Anderson, D.C. Licciardello, T.V. Ramakrishnan, Phys. Rev. Lett. 42, 673 (1979)

6. S. Sachdev, Quantum Phase Transitions (Cambridge University Press, Cambridge, 2011)

7. D.S. Fisher, Phys. Rev. Lett. 69, 534 (1992)

8. D.S. Fisher, Phys. Rev. B 51, 6411 (1995)

9. G. Tarjus, M. Tissier, Eur. Phys. J. B 93, 50 (2020)

10. M. Kumar, F. Corberi, E. Lipiello, S. Puri, Eur. Phys. J. B 93, 88 (2020)

11. G. Delfino, N. Lamsen, Eur. Phys. J. B 92, 278 (2019)

12. S. Cocco, G. Croce, F. Zamponi, Eur. Phys. J. B 92, 259 (2019)

13. R. Kumar, J. Gross, W. Janke, M. Weigel, Eur. Phys. J. B 93, 79 (2020)

14. S. Schnabel, W. Janke, Eur. Phys. J. B 93, 53 (2020)

15. Z.-L. Tsai, P. Chen, Y.-C. Lin, Eur. Phys. J. B 93, 63 (2020)

16. V.L. Quito, P.L.S. Lopes, J.A. Hoyos, E. Miranda, Eur. Phys. J. B 93, 17 (2020)

17. J.C. Getelina, J.A. Hoyos, Eur. Phys. J. B 93, 2 (2020)

18. C. Zhang, H. Rieger, Eur. Phys. J. B 93, 25 (2020)

19. G. Roósz, I.A. Kovács, F. Iglói, Eur. Phys. J. B 93, 8 (2020)

20. K. Slevin, S. Kettemann, T. Ohtsuki, Eur. Phys. J. B 92, 281 (2019)

21. H. Eleuch, M. Hilke, Eur. Phys. J. B 92, 269 (2019)

22. K-S. Kim, J-H. Han, Eur. Phys. J. B 93, 21 (2020)

23. S.J. Thomson, M. Schiró, Eur. Phys. J. B 93, 22 (2020)

24. N.C. Chávez, F. Mattiotti, J.A. Méndez-Bermúdez, F. Borgonovi, G.L. Celardo, Eur. Phys. J. B 92, 144 (2019)

25. S.E. Skipetrov, Eur. Phys. J. B 93, 70 (2020)

26. J.M. Luck, Eur. Phys. J. B 92, 180 (2019)

27. C. Monthus, Eur. Phys. J. B 92, 149 (2019) 